ARTICLE

Received 9 May 2014 | Accepted 17 Oct 2014 | Published 27 Nov 2014

DOI: $10.1038 /$ ncomms 6584

\title{
Observing optical coherence across Fock layers with weak-field homodyne detectors
}

\author{
Gaia Donati ${ }^{1}$, Tim J. Bartley ${ }^{1}$, Xian-Min Jin ${ }^{1,2}$, Mihai-Dorian Vidrighin ${ }^{1,3}$, Animesh Datta1,
} Marco Barbieri ${ }^{1,4} \&$ Ian A. Walmsley ${ }^{1}$

Quantum properties of optical modes are typically assessed by observing their photon statistics or the distribution of their quadratures. Both particle- and wave-like behaviours deliver important information and each may be used as a resource in quantum-enhanced technologies. Weak-field homodyne (WFH) detection provides a scheme that combines the wave- and particle-like descriptions. Here we show that it is possible to observe a wave-like property such as the optical coherence across Fock basis states in the detection statistics derived from discrete photon counting. We experimentally demonstrate these correlations using two WHF detectors on each mode of two classes of two-mode entangled states. Furthermore, we theoretically describe the response of WHF detection on a two-mode squeezed state in the context of generalized Bell inequalities. Our work demonstrates the potential of this technique as a tool for hybrid continuous/discrete-variable protocols on a phenomenon that explicitly combines both approaches.

\footnotetext{
${ }^{1}$ Clarendon Laboratory, University of Oxford, Parks Road, Oxford OX1 3PU, UK. ${ }^{2}$ Department of Physics, Shanghai Jiao Tong University, Shanghai 200240 China. ${ }^{3}$ QOLS, Blackett Laboratory, Imperial College London, London SW7 2BW, UK. ${ }^{4}$ Dipartimento di Scienze, Università degli Studi Roma Tre, Via della Vasca Navale 84, 00146 Rome, Italy. Correspondence and requests for materials should be addressed to G.D. (email: g.donati1@physics.ox.ac.uk).
} 
Q uantum correlations play a central role in our understanding of fundamental quantum physics and represent a key resource for quantum technologies ${ }^{1-4}$. Progress in quantum information science has followed an increasingly thorough understanding of how such correlations manifest themselves and how they can be successfully generated, manipulated and characterized ${ }^{5}$. In quantum optical systems, these correlations appear in either properties of the fields, such as quadrature entanglement, or at the level of individual quanta, that is, photons. Access and control over such correlations are key to applications in quantum information science; however, how the complementary mode and particle correlations precisely act as a resource is still a subject of debate ${ }^{6}$. The capability of studying the correlations in a regime traversing mode and particle aspects is thus necessary for clarifying the origin of quantum enhancement.

When a coherent superposition of many photons occupies a single mode, a wave-like description of the quantum state in terms of continuous variables (that is, the values of the quadratures) of the electromagnetic field is the standard approach ${ }^{7}$. The canonical technique for measuring such light fields is strong-field homodyne detection, which directly probes the quadratures of a field and can provide a full reconstruction of its quantum state ${ }^{8}$. On the other hand, particle-like properties can be directly accessed with a range of photodetectors, a notable example being photon-number-resolving detectors (PNRDs) ${ }^{9-12}$, and suitable techniques for the reconstruction of the photon statistics $^{13,14}$. However, such photon counters are intrinsically insensitive to phase, and thus cannot access any coherence between modes. Weak-field homodyne (WFH) has been proposed as a measurement technique bridging wave- and particle-like descriptions ${ }^{15-21}$. As in standard homodyne, a local oscillator (LO) provides phase sensitivity, while the photon statistics are accessed by number-resolving detectors. The main difference is that WFH makes use of a classical reference whose mean photon number is of a similar order as that of the probed signal; this allows for the combination of the homodyne technique with PNRDs based on photon counting modules.

In this paper, we employ WFH detection to investigate coherence between different photon-number basis states (Fock layers) across two-mode entangled states. Our detection scheme accesses this manifestation of optical coherence directly, without the need for resource-intensive full state tomography. We demonstrate the oscillations of an array of multi-photon coincidence counts when a split single-photon state (SSPS) and a two-mode squeezed state (TMSS) are interfered with a weak LO. Our experiment can be regarded as a first step towards the quantitative study of the nonlocal properties of multi-mode quantum states with multi-outcome measurements by nonGaussian detectors. To this end, we theoretically study a violation of Bell inequalities that can be achieved for low numbers of detected photons under ideal conditions.

\section{Results}

Experiment. The WFH setup is depicted in Fig. 1a. A signal is mixed on a beam splitter with a local oscillator, described by the coherent state $\left|\alpha e^{i \phi}\right\rangle$, which establishes a phase reference. One or both output modes from the beam splitter are then detected using PNRDs. The method is termed 'weak-field' since the intensity of the LO is comparable to that of the signal. This differs from strong-field homodyne in which the coherent state is many orders of magnitude more intense than the signal, and the outputs are detected by linear photodiodes.

To understand how WFH can detect coherence across Fock layers, consider the detection of $m$ photons. These can originate from any Fock term $|k\rangle$ in the signal together with $m-k$ photons from the LO; if the complex coefficients of the terms $\{|k\rangle\}$ have well-defined relative phases, then these result in a modulation of the detection probability $p(m)$ as the phase of the LO is varied. For a two-mode state, the relevant phases are the ones between joint detection events coming from terms in the signal of the form $\left|k_{1}\right\rangle\left|k_{2}\right\rangle$. Consequently, coherence across such events can be observed in the modulation of joint detection probabilities.

We use the layout shown in Fig. 1b to study the coherence between Fock layers across the two modes of a split single photon and a squeezed vacuum state. To maximize the passive phase stability of our setup, we adopt a compact design in which the LO and the signal are collinear and occupy two orthogonal polarizations of a single spatial mode. Our PNRDs are timemultiplexed detectors (TMDs) that split an incoming beam into two spatial and four temporal modes, thereby resolving up to eight photons probabilistically using two avalanche photodiodes $^{10,22}$. Specifically, the TMDs allow us to decompose the intensity of the interference patterns resulting from each output mode into its constituent photon components. In this way, we can probe pair-wise correlations between the individual Fock layers as we build up a joint detection statistics matrix, every row and column representing the number of clicks in each detected mode. The click statistics gives us access to higher-order Fock states $(k \geq 1)$, although this detection scheme is not fully equivalent to a number-resolving detector ${ }^{23}$.

A split single-photon state may be written as

$$
|\Psi\rangle_{\mathrm{SSPS}}=\frac{1}{\sqrt{2}}\left(|0\rangle_{\mathrm{A}}|1\rangle_{\mathrm{B}}+|1\rangle_{\mathrm{A}}|0\rangle_{\mathrm{B}}\right)
$$

which describes a single photon in a coherent superposition of modes A and B (see Fig. 2a and Supplementary Fig. 1 for further details on the experimental layout). The results of our investigation for the SSPS are reported in Fig. 3.
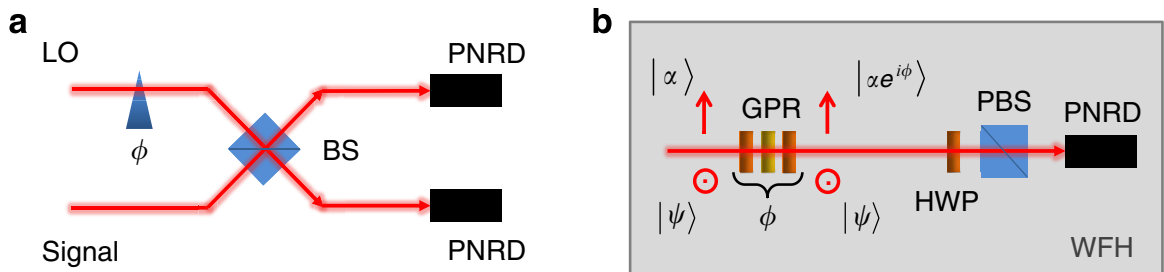

Figure 1 | Layout of the WFH detector. (a) The general scheme relies on the interference between a signal and a LO of similar intensity for different settings of the phase $\phi$. Either one or both outputs are measured with PNRDs. (b) Our experimental implementation adopts a collinear configuration in which the signal $|\psi\rangle$ and the LO have orthogonal polarizations. For this reason, the beam splitter in a is replaced by a half-wave plate (HWP) and a PBS that realize interference in polarization. The phase setting of the LO is controlled by means of a geometric phase rotator (GPR), which consists of a quarter-wave plate (QWP), a HWP and a second QWP. The rotation of the HWP (with both QWP fixed at $45^{\circ}$ ) applies a phase shift to the coherent state. A TMD records click events on the transmitted output mode. 
The red dots show the experimental probabilities $P\left(m, m^{\prime}\right)$ of the joint detection of $m$ clicks in mode A and $m^{\prime}$ clicks in mode B. Each $P\left(m, m^{\prime}\right)$ term is a function of the difference between the phase settings of the weak LOs, $\phi^{(-)}=\phi_{\mathrm{A}}-\phi_{\mathrm{B}}$, since a single photon has no phase per $s e^{24,25}$. Our count rates are such that we only consider events where $m, m^{\prime} \leq 2$; detection outcomes greater than this level are negligible. The blue lines are predictions from a model that accounts for the following imperfections in our experiment: non-unit efficiency of the detectors; modulation of the reflectivities of the beam splitters preceding the TMDs when varying the LO phases (due to the geometric phase rotators depicted in Fig. 1b-see Methods and Supplementary Fig. 2). We a

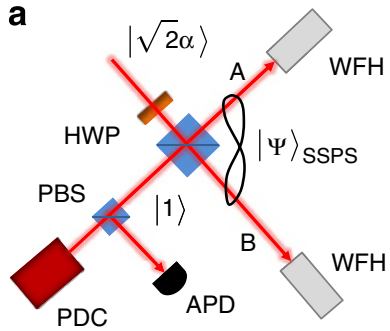

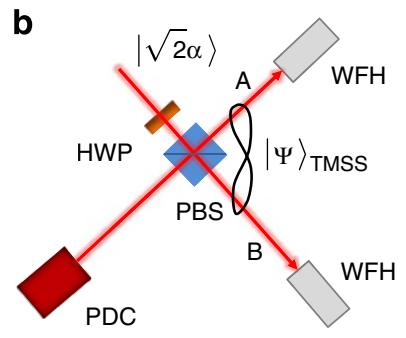

Figure 2 | Probing correlations across optical modes with WFH. (a) A single photon is produced by a heralded source based on parametric downconversion. We then split the single-photon state to generate $|\Psi\rangle_{\text {SSPS }}$ across two separate spatial modes ( $A$ and $B$ ), both probed by the WFH detectors described in Fig. 1b. (b) The same downconversion source produces the squeezed vacuum state whose two modes are also probed by WFH detectors. also include imperfections in the production of the single-photon states $^{26}$, and we thus write

$$
\rho_{0}=w_{0}|0\rangle\left\langle 0\left|+w_{1}\right| 1\right\rangle\left\langle 1\left|+\left(1-w_{0}-w_{1}\right)\right| 2\right\rangle\langle 2|,
$$

where $w_{0}$ and $w_{1}$ are coefficients taken between 0 and 1 that weight the zero- and one-photon contributions to the input state. We experimentally determine these parameters from the photon statistics of the initial state (see Supplementary Note 1 and Supplementary Note 2).

The experimental curves show oscillations in the coincidence counts: these are evident in the $P(1,1), P(2,1)$ and $P(1,2)$ terms, as also predicted by the theory. What is most striking about the observed oscillatory behaviour is the fact that it is displayed by terms $P\left(m, m^{\prime}\right)$ for which $m+m^{\prime}>1$, yet it is determined by the coherence between the terms $|0\rangle_{\mathrm{A}}|1\rangle_{\mathrm{B}}$ and $|1\rangle_{\mathrm{A}}|0\rangle_{\mathrm{B}}$ (see equation (1)), which do not contain more than one photon each. Ideally, any additional photon detected must therefore come from the LOs, hence the SSPS is responsible for the coherent oscillations observed in the considered joint detection probabilities. In practice, we observe good qualitative agreement between the experimental data and our theoretical description. Indeed, we are able to account for all the main features of a 3-by-3 array of multi-photon counts with one model that has no free parameters; detection efficiencies and the weights in equation (2) are experimentally determined. We attribute the residual discrepancies to two main factors: imperfect mode matching between the LOs and the signal modes and variations of the laser power during the few-hour-long acquisition.

An analogous experiment is conducted on a TMSS (see Fig. 2b and Supplementary Fig. 3 for further details on the experimental layout). This state is archetypal of a quantum resource with
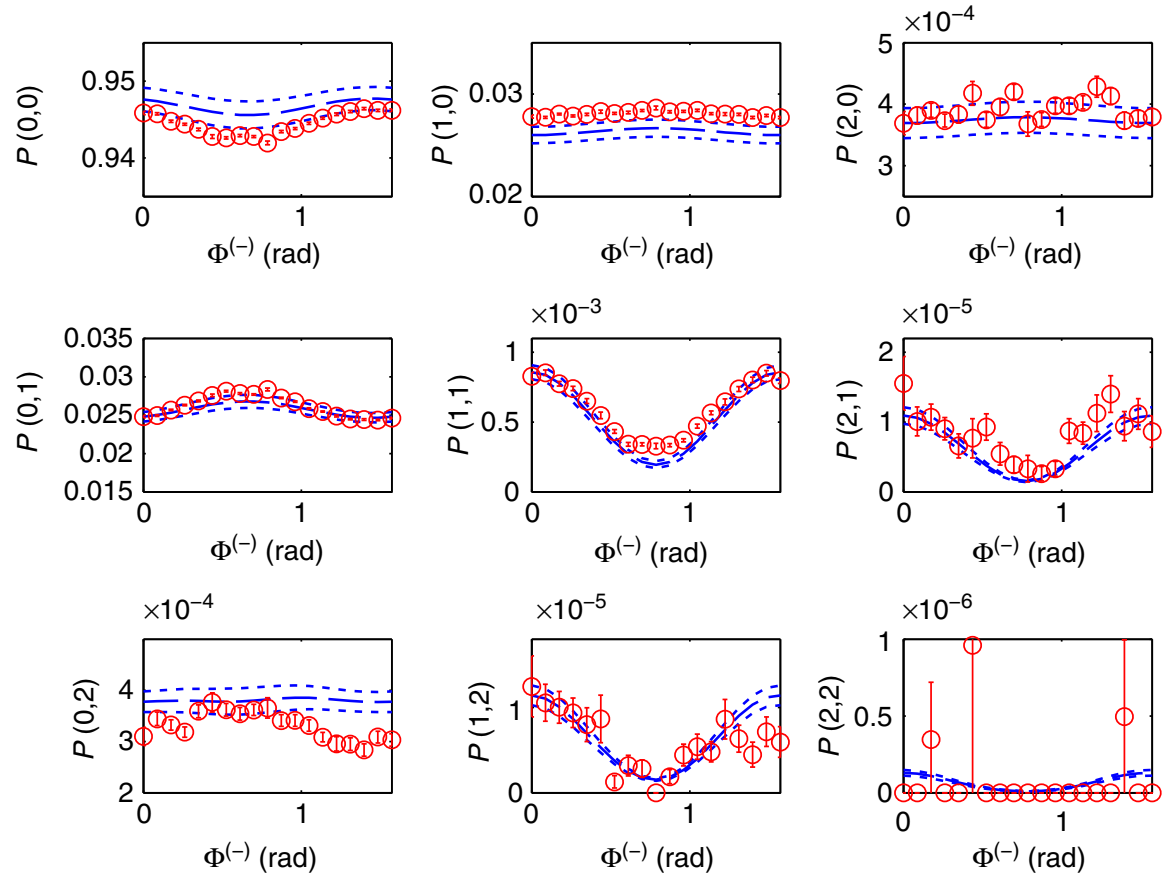

Figure 3 | Joint counting statistics for the SSPS. Correlations between the responses of two WFH detectors as a function of the phase difference $\Phi^{(-)}=\phi^{(-)} / 4$ (where $\Phi^{(-)}$is determined by the settings of the half-wave plates in the phase rotators). We recall that $P\left(m, m^{\prime}\right)$ is associated to the joint detection probability of $m$ photons on mode $\mathrm{A}$ and $\mathrm{m}^{\prime}$ photons on mode $\mathrm{B}$. The red dots are measured probabilities, with uncertainties determined by a Monte Carlo routine under the assumption of a Poissonian distribution for the raw counts. The blue theoretical curves are obtained from a model that includes the main imperfections in the setup. The dashed curves correspond to an input state with the experimentally determined weights $w_{0}=0.161$ and $w_{1}=0.669$. The uncertainties $\sigma_{0}=0.011$ on $w_{0}$ and $\sigma_{1}=0.029$ on $w_{1}$ are estimated by a Monte Carlo routine; the dotted curves show the results given by the theoretical model with $\left(w_{0}-\sigma_{0}, w_{1}-\sigma_{1}\right)$ and $\left(w_{0}+\sigma_{0}, w_{1}+\sigma_{1}\right)$, respectively. The quantum efficiencies of the detectors, $\eta_{\mathrm{A}}=0.072$ and $\eta_{\mathrm{B}}=0.064$, are experimentally estimated with the Klyshko method ${ }^{44,45}$. The intensities of the two local oscillators are $\left|\alpha_{A}\right|=0.510$ and $\left|\alpha_{B}\right|=0.585$. 

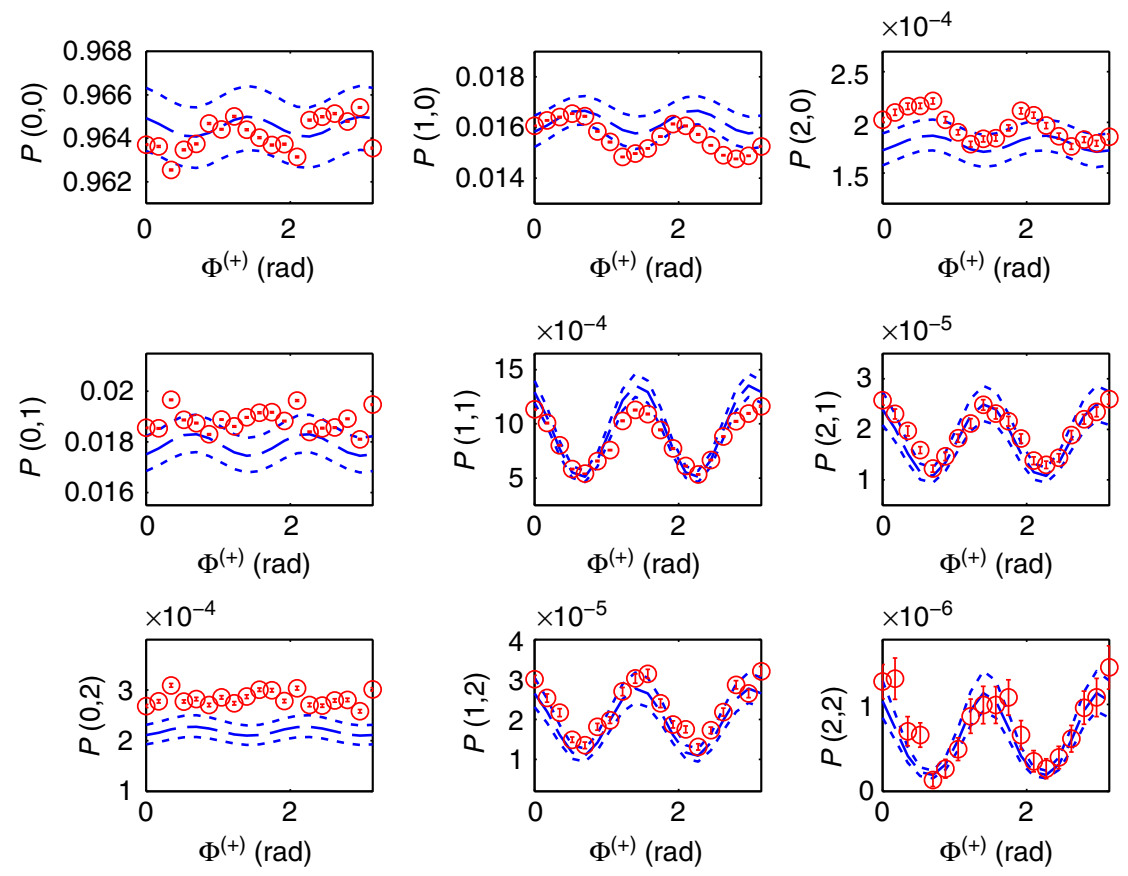

Figure 4 | Joint counting statistics for the TMSS. Correlations between the responses of two WFH detectors as a function of the phase sum $\Phi^{(+)}=\phi^{(+)} / 4$ (where $\Phi^{(+)}$is determined by the settings of the half-wave plates in the phase rotators). We recall that $P\left(m, m^{\prime}\right)$ is associated to the joint detection probability of $m$ photons on mode $A$ and $m^{\prime}$ photons on mode $B$. The red dots are measured probabilities, with uncertainties determined by a Monte Carlo routine under the assumption of a Poissonian distribution for the raw counts. The blue theoretical curves are obtained from a model that includes the main imperfections in the setup. The dashed curves correspond to an input state with the experimentally determined squeezing parameter $\lambda=0.295$ and weight $P=0.04$ for the additional noise term. The uncertainty $\sigma=0.016$ on $\lambda$ is estimated by a Monte Carlo routine; the dotted curves show the results given by the theoretical model with $\lambda \pm \sigma$. The experimental quantum efficiencies of the detectors are $\eta_{\mathrm{A}}=0.132$ and $\eta_{\mathrm{B}}=0.155$ (estimated with the Klyshko method). The intensities of the two local oscillators are $\left|\alpha_{A}\right|=0.365$ and $\left|\alpha_{B}\right|=0.347$.
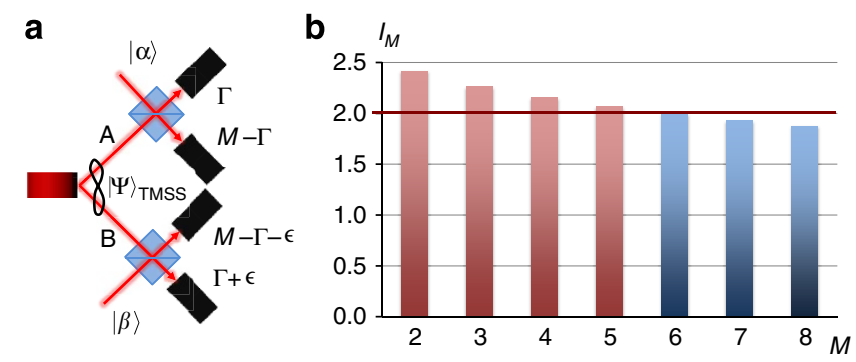

Figure 5 | Generalized Bell inequalities with WFH. (a) In the ideal case, the two modes of a TMSS are interfered with two weak LOs; PNRDs monitor all four outputs. We fix the number of detected photons to $M$ on each side and label the various detection events recorded by the PNRDs according to how the $M$ particles distribute themselves on the two output modes: $\Gamma, M-\Gamma, \Gamma+\epsilon$ and $M-\Gamma-\epsilon(\mathbf{b})$ The plot shows the values taken by the quantity $I_{M}$ (see equation (5)) when the number of detected photons $M$ on each side varies between two and eight. The bound for $I_{M}$ predicted by local realism is two for all values of $M$, hence we see that no violation of a generalized Bell inequality is possible beyond $M=5$.

well-known correlations between its Fock layers. The expression for a TMSS reads

$$
|\Psi\rangle_{\mathrm{TMSS}}=\sqrt{1-\lambda^{2}} \sum_{n=0}^{+\infty} \lambda^{n}|n\rangle_{\mathrm{A}}|n\rangle_{\mathrm{B}}
$$

where the real squeezing parameter $\lambda$ governs the photon distribution across the photon-number basis states. Here, we pump our source with moderate parametric gain to generate significant higher-order Fock layers in the two-mode state ${ }^{27}$. The expected phase dependence of $\left\{P\left(m, m^{\prime}\right)\right\}$ for a TMSS was shown to be $\phi^{(+)}=\phi_{\mathrm{A}}+\phi_{\mathrm{B}} \quad$ (ref. 28). The TMSS has a phase dependence arising from that of the pump; in a conventional strong homodyne setup this would define which quadratures are squeezed. In our case, the same effect is manifested in the phase dependence of the click patterns; hence, appropriate phase locking was necessary (see Methods).

The results obtained for the TMSS are illustrated in Fig. 4. Once again, we observe good qualitative agreement between our data and the predictions from a theoretical model that includes the same imperfections as for the single-photon case. Here the input state can be modelled as

$$
\rho=(1-p)|\Psi\rangle_{\mathrm{TMSS}}\langle\Psi|+p| 0,1\rangle\langle 0,1|,
$$

where the extra term is a first-order approximation of noise in a squeezed thermal state. This asymmetry across the modes is justified by the experimentally recorded $g^{(2)}$ for the parametric downconversion source: we find $g_{\mathrm{A}}^{(2)}=1.98 \pm 0.04$ and $g_{\mathrm{B}}^{(2)}=$ $1.92 \pm 0.04$ for the two marginals, to be compared with $g^{(2)}=2$ for an ideal thermal state. The lower value of $g_{\mathrm{B}}^{(2)}$ motivates the addition of the noise term on output mode B. The quoted values of $g^{(2)}$ also suggest that additional (and undesired) Schmidt modes might be responsible for the presence of photons in modes correlated to undetected modes ${ }^{29}$. As for the case of the SSPS, imperfect mode matching between the LOs and the signal modes and variations of the laser power during the data acquisition are recognized as the main causes of the residual departures of the experimental curves from the expected behaviour in Fig. 4.

As a general remark applying to both classes of studied states, we note that our numerical models depend strongly 
on the detection efficiencies (see Supplementary Figs 4 and 5). In this sense, the more pronounced discrepancies in Figs 3 and 4, such as that in the $P(0,2)$ terms, may be due to noise affecting our estimation of these parameters. More details on the theoretical model for both the SSPS and the TMSS can be found in Supplementary Notes 1 and 2.

Generalized Bell inequalities for WFH detection. Correlations such as those revealed in our experiment need to be understood in relation to canonical criteria for non-classicality, for instance the violation of a Bell inequality, to assess their role as possible quantum resources ${ }^{30}$. The ability to access and discriminate higher photon numbers leads us to refer to generalized, higherdimensional Bell inequalities ${ }^{31}$, which we study theoretically in the context of our experiment with a TMSS.

To this end, let us consider the scenario depicted in Fig. 5. Each mode of a TMSS is analysed by means of a WFH detector; here all four outputs are monitored by perfect PNRDs. We take into account the Fock layers that lead to the detection of $M$ photons on each side: the detected photons are split among the two output ports of the polarizing beam splitters (PBSs) (that is, the photons are either transmitted or reflected) according to the convention indicated in Fig. 5a. Specifically, we consider measurements with $D=M+1$ possible results, distinguished according to the number of photons $\Gamma$ detected on the transmitted arm of each PBS. We are thus interested in the probabilities associated with joint detection events comprising outcomes (on each output arm, on both sides) differing by $\epsilon$ (the outcomes being taken modulo $D) .\left\{\alpha, \alpha^{\prime}\right\}$ and $\left\{\beta, \beta^{\prime}\right\}$ denote the LO settings on side A and side B, respectively. The relevant probabilities are combined into the expression

$$
\begin{aligned}
I_{M} & =\sum_{\epsilon=0}^{\left[\frac{M+1}{2}\right]-1}\left(1-\frac{2 \epsilon}{M}\right)\left[P(\alpha, \beta, \epsilon)+P\left(\alpha^{\prime}, \beta,-\epsilon-1\right)\right. \\
& \left.+P\left(\alpha^{\prime}, \beta^{\prime}, \epsilon\right)+P\left(\alpha, \beta^{\prime},-\epsilon\right)\right]-[P(\alpha, \beta,-\epsilon-1) \\
& \left.+P\left(\alpha^{\prime}, \beta, \epsilon\right)+P\left(\alpha^{\prime}, \beta^{\prime},-\epsilon-1\right)+P\left(\alpha, \beta^{\prime}, \epsilon+1\right)\right],
\end{aligned}
$$

where the local realistic bound is $\left|I_{M}\right| \leq 2$ (ref. 31). We note that the case $M=1$ corresponds to the standard CHSH inequality that was experimentally tested in ref. 17. Additional details on how to compute $I_{M}$ for the specific layout that we consider are provided in Supplementary Note 3, Supplementary Figs 6 and 7.

We run a numerical search for values of $M$ ranging from 2 to 8 to find the set of parameters $\left\{\lambda, \alpha, \alpha^{\prime}, \beta, \beta^{\prime}\right\}$ that determine the highest violation of these generalized Bell inequalities. Our results are shown in Fig. 5b: no violation of equation (5) is found beyond $M=5$. This behaviour comes from the fact that the maximal violation of equation (5) (that is, the attainment of the maximum value allowed by quantum mechanics) relies on a particular structure for the entangled state ${ }^{32}$. Notably, a TMSS is specified solely by the squeezing parameter $\lambda$ : this means that there are not enough degrees of freedom to tune to obtain the required form for the input state. This restriction becomes more severe as the dimensionality of the system increases, to the point that no violation of local realism can be inferred despite the use of an entangled resource such as a TMSS. Hence the decrease in the violation stems from the Gaussian character of the TMSS, which fixes the functional shape of the oscillation curves simultaneously for all Fock layers.

\section{Discussion}

We have shown how WFH can be used to deconstruct phasedependent measurements on two-mode entangled states into their constituent Fock layers. The ability to operate devices at the interface of wave and particle regimes opens up new possibilities for quantum information processing ${ }^{33}$. Thus, our work provides a new insight on such resources within the broader investigations on hybrid continuous/discrete-variable coding ${ }^{34-38}$.

As a complement, we have also studied the theory of the violation of generalized Bell inequalities using an ideal WFH setup. These tests shed light on how the transition from nonGaussian to Gaussian measurements occurs. Such transition is achieved in the context of WFH detection as an increase in the LO strength. In fact, this study may also be interpreted in the more general framework of Gaussian versus non-Gaussian measurements, where it is well-known that the outcomes of a Gaussian measurement on a Gaussian quantum state can be explained by a local realistic model. For this reason, strong-field homodyne detection on a TMSS cannot be used to violate a Bell inequality ${ }^{39,40}$. On the other hand, weak-field homodyne is an example of non-Gaussian measurement, as attested by its Wigner function. Consequently, we expect that the outcomes of WFH detection on an entangled Gaussian state cannot be described by a local realistic model ${ }^{17,41,42}$; however, this breaks for moderately high photon numbers.

On the experimental side, further developments for the observation of the violation of higher-dimensional Bell inequalities demand detectors with higher quantum efficiency ${ }^{43}$. These are not only necessary for achieving significant counting statistics, but also to prevent one Fock layer to be affected from higherorder contributions. Encouraging results have been obtained in this direction with cryogenic detectors.

\section{Methods}

Source of quantum states. A pulsed Ti:Sapphire laser (repetition rate $256 \mathrm{kHz}$ central wavelength $\lambda_{\mathrm{IR}}=830 \mathrm{~nm}$ and bandwidth $\Delta \lambda_{\mathrm{IR}} \simeq 30 \mathrm{~nm}$ ) is doubled in a nonlinear crystal ( $\beta$-barium borate). The second-harmonic beam $\left(\lambda_{\mathrm{UV}}=415 \mathrm{~nm}\right)$ pumps a type-II parametric downconversion process in a nonlinear crystal (potassium dihydrogen phosphate) to produce a two-mode vacuum-squeezed state. The source is designed to generate spectrally uncorrelated modes based on group velocity mismatch inside the birefringent medium ${ }^{27}$. Daughter photons have orthogonal polarizations and different spectral widths $\left(\Delta \lambda_{\mathrm{V}} \simeq 12 \mathrm{~nm}, \Delta \lambda_{\mathrm{H}} \simeq 6 \mathrm{~nm}\right)$. The same source generates the SSPS and the TMSS depending on the ultraviolet pump beam brightness.

WFH detection. The detection system that we adopt in our experiment is realized with a collinear geometry to ensure passive phase stability. Each mode in equations (1) and (3) is superposed with a LO of orthogonal polarization at a PBS. This delivers a common spatial mode at the output but orthogonal polarizations for the signal to be probed and the weak coherent beam. Interference is then realized by a half-wave plate and an additional PBS. The relative phase between the horizontal and vertical polarizations can be adjusted by means of a geometric phase rotator (GPR-see Fig. 1b), which is composed of a quarter-wave plate (QWP), a half-wave plate and a second QWP. The axis of the first QWP is set to $45^{\circ}$ to transform the input linear polarizations into circular ones. The rotation of the HWP by an angle $\phi / 4$ results in a phase shift equal to $\phi$ between left- and right-circular polarizations The initial linear polarizations are then recovered by setting the second QWP to $45^{\circ}$. The successful calibration of the full device relies on the characterization of each element (including the PBS), particularly of the QWPs. When the GPR is correctly calibrated power fluctuations around $3 \%$ are recorded, while imperfections in the calibration of one of its constituents can lead to fluctuations in power above $10 \%$ (see Supplementary Fig. 2). Finally, the output state is analysed with a TMD: this consists of two fibre-based cascaded Mach-Zehnder interferometers that split the incoming state over two spatial modes and four distinct time bins. Time-resolved clicks from avalanche photodiodes monitoring the two transmitted modes are thus registered.

Active phase stabilization. To actively lock the phase set by the GPRs, we use an ancillary laser beam (from a continuous-wave HeNe laser, $\lambda^{\prime}=633 \mathrm{~nm}$ ), which back-propagates through the interferometer. The classical interference pattern that we obtain when the ancillary beam reproduces correctly the signal and LO optical paths constitutes the signal recorded by a photodiode connected to a PID device (SRS SIM960 Analog PID Controller). 


\section{References}

1. Schrödinger, E. Die gegenwärtige Situation in der Quantenmechanik. Naturwissenschaften 23, 844-849 (1935).

2. Wiseman, H. M., Jones, S. J. \& Doherty, A. C. Steering, entanglement, nonlocality, and the Einstein-Podolsky-Rosen paradox. Phys. Rev. Lett. 98, 140402 (2007).

3. Modi, K., Brodutch, A., Cable, H., Paterek, T. \& Vedral, V. The classicalquantum boundary for correlations: discord and related measures. Rev. Mod. Phys. 84, 1655-1707 (2012).

4. Silva, I. A. et al. Measuring bipartite quantum correlations of an unknown state. Phys. Rev. Lett. 110, 140501 (2013).

5. Baumgratz, T., Cramer, M. \& Plenio, M. B. Quantifying coherence. Phys. Rev. Lett. 113, 140401 (2014).

6. Demkowicz-Dobrzański, R., Jarzyna, M. \& Kolodyński, J. Quantum limits in optical interferometry. Preprint at http://arxiv.org/abs/1405.7703v2 (2014).

7. Braunstein, S. L. \& Van Loock, P. Quantum information with continuous variables. Rev. Mod. Phys. 77, 513-577 (2005).

8. Lvovsky, A. I. \& Raymer, M. G. Continuous-variable optical quantum-state tomography. Rev. Mod. Phys. 81, 299-332 (2009).

9. Divochiy, A. et al. Superconducting nanowire photon-number-resolving detector at telecommunication wavelengths. Nat. Photonics 2, 302-306 (2008).

10. Achilles, D. et al. Photon-number-resolving detection using time-multiplexing. J. Mod. Opt. 51, 1499-1515 (2009).

11. Jahanmirinejad, S. et al. Photon-number resolving detector based on a series array of superconducting nanowires. Appl. Phys. Lett. 101, 072602 (2012).

12. Calkins, B. et al. High quantum-efficiency photon-number-resolving detector for photonic on-chip information processing. Opt. Express 21, 22657-22670 (2013)

13. Zambra, G. et al. Experimental reconstruction of photon statistics without photon counting. Phys. Rev. Lett. 97, 063602 (2005).

14. Allevi, A., Olivares, S. \& Bondani, M. Measuring high-order photon-number correlations in experiments with multimode pulsed quantum states. Phys. Rev. A 85, 063835 (2012)

15. Wallentowitz, S. \& Vogel, W. Unbalanced homodyning for quantum state measurements. Phys. Rev. A. 53, 4528 (1996).

16. Banaszek, K., Radzewicz, C., Wódkiewicz, K. \& Krasiński, J. S. Direct measurement of the Wigner function by photon counting. Phys. Rev. A 60, 674 (1999).

17. Kuzmich, A., Walmsley, I. A. \& Mandel, L. Violation of Bell's inequality by a generalized Einstein-Podolsky-Rosen state using homodyne detection. Phys. Rev. Lett. 85, 1349 (2000)

18. Resch, K. J., Lundeen, J. S. \& Steinberg, A. M. Quantum state preparation and conditional coherence. Phys. Rev. Lett. 88, 113601 (2002).

19. Puentes, G. et al. Bridging particle and wave sensitivity in a configurable detector of positive operator-valued measures. Phys. Rev. Lett. 102, 080404 (2009).

20. Laiho, K., Cassemiro, K. N., Gross, D. \& Silberhorn, C. Probing the negative Wigner function of a pulsed single photon point by point. Phys. Rev. Lett. 105, 253603 (2010)

21. Zhang, L. et al. Mapping coherence in measurement via full quantum tomography of a hybrid optical detector. Nat. Photonics 6, 364-368 (2012).

22. Lundeen, J. S. et al. Tomography of quantum detectors. Nat. Phys. 5, 27-30 (2009).

23. Sperling, J., Vogel, W. \& Agarwal, G. S. Sub-binomial light. Phys. Rev. Lett. 109, 093601 (2012)

24. Babichev, S. A., Brezger, B. \& Lvovsky, A. I. Remote preparation of a singlemode photonic qubit by measuring field quadrature noise. Phys. Rev. Lett. 92, 047903 (2004)

25. Morin, O. et al. Witnessing trustworthy single-photon entanglement with local homodyne measurements. Phys. Rev. Lett. 110, 130401 (2013).

26. Bartley, T. J. et al. Multiphoton state engineering by heralded interference between single photons and coherent states. Phys. Rev. A 86, 043820 (2012).

27. Mosley, P. J. et al. Heralded generation of ultrafast single photons in pure quantum states. Phys. Rev. Lett. 100, 133601 (2008).

28. Blandino, R. et al. Homodyne estimation of Gaussian quantum discord. Phys. Rev. Lett. 109, 180402 (2012).

29. Mosley, P. J., Lundeen, J. S., Smith, B. J. \& Walmsley, I. A. Conditional preparation of single photons using parametric downconversion: a recipe for purity. New J. Phys. 10, 093011 (2008)
30. Brunner, N., Cavalcanti, D., Pironio, S., Scarani, V. \& Wehner, S. Bell nonlocality. Rev. Mod. Phys. 86, 419-478 (2014)

31. Collins, D., Gisin, N., Linder, N., Massar, S. \& Popescu, S. Bell inequalities for arbitrarily high-dimensional systems. Phys. Rev. Lett. 88, 040404 (2002).

32. Acín, A., Durt, T., Gisin, N. \& Latorre, J. I. Quantum nonlocality in two threelevel systems. Phys. Rev. A 65, 052325 (2001).

33. Kiesel, T., Vogel, W., Hage, B. \& Schnabel, R. Entangled qubits in a nonGaussian quantum state. Phys. Rev. A 83, 062319 (2011).

34. Ourjoumtsev, A., Ferreyrol, F., Tualle-Brouri, R. \& Grangier, P. Preparation of non-local superpositions of quasi-classical light states. Nat. Phys 5, 189-192 (2009).

35. Neergaard-Nielsen, J. S. et al. Optical continuous-variable qubit. Phys. Rev. Lett. 105, 053602 (2010)

36. Neergaard-Nielsen, J. S., Eto, Y., Lee, C.-W., Jeong, H. \& Sasaki, M. Quantum tele-amplification with a continuous-variable superposition state. Nat. Photonics 7, 439-443 (2013).

37. Jeong, H. et al. Generation of hybrid entanglement of light. Nat. Photonics $\mathbf{8}$, 564-569 (2014)

38. Morin, O. et al. Remote creation of hybrid entanglement between particle-like and wave-like optical qubits. Nat. Photonics 8, 570-574 (2014).

39. Gilchrist, A., Deuar, P. \& Reid, M. D. Contradiction of quantum mechanics with local hidden variables for quadrature phase amplitude measurements. Phys. Rev. Lett. 80, 3169 (1998).

40. García-Patrón, R. et al. Proposal for a loophole-free Bell test using homodyne detection. Phys. Rev. Lett. 93, 130409 (2004).

41. Björk, G., Jonsson, P. \& Sánchez-Soto, L. L. Single-particle nonlocality and entanglement with the vacuum. Phys. Rev. A 64, 042106 (2001).

42. Hessmo, B., Usachev, P., Heydari, H. \& Björk, G. Experimental demonstration of single photon nonlocality. Phys. Rev. Lett. 92, 180401 (2004).

43. Eisaman, M. D., Fan, J., Migdall, A. \& Polyakov, S. V. Single-photon sources and detectors. Rev. Sci. Instrum. 82, 071101 (2011)

44. Klyshko, D. N. Observable signs of nonclassical light. Phys. Lett. A 213, 7-15 (1996).

45. Achilles, D., Silberhorn, C. \& Walmsley, I. A. Direct, loss-tolerant characterization of nonclassical photon statistics. Phys. Rev. Lett. 97, 043602 (2006).

\section{Acknowledgements}

We are grateful to J. Spring and A. Eckstein for helping with the detection setup, and thank L. Zhang, B. Smith, M.S. Kim and M. Paternostro for fruitful discussions. This work was supported by the EPSRC (EP/K034480/1, EP/H03031X/1, EP/H000178/1), the EC project SIQS and the Royal Society. X.-M.J. acknowledges support from the Nationa Natural Science Foundation of China (Grant No. 11374211) and an EC Marie Curie fellowship (PIIF-GA-2011-300820). A.D. is supported by the EPSRC (EP/K04057X/1).

\section{Author contributions}

G.D. performed the experiments and, with assistance from A.D., M.B. and M.-D.V., analysed the data and investigated the application of WFH to generalized Bell inequalities. G.D. and T.J.B., with assistance from M.B., X.-M.J. and M.-D.V., designed the experimental layout. T.J.B., M.B. and I.A.W. conceived the experiment. G.D., T.J.B., A.D. and M.B. wrote the paper with contributions from all authors. All authors agree to the contents of the article.

\section{Additional information}

Supplementary Information accompanies this paper at http://www.nature.com/ naturecommunications

Competing financial interests: The authors declare no competing financial interest.

Reprints and permission information is available online at http://npg.nature.com/ reprintsandpermissions/

How to cite this article: Donati, G. et al. Observing optical coherence across Fock layer with weak-field homodyne detectors. Nat. Commun. 5:5584 doi: 10.1038/ncomms6584 (2014). 